\title{
Scale Hierarchies in Particle Physics and Cosmology
}

\author{
I. Antoniadis ${ }^{1,2, a}$ \\ ${ }^{1}$ LPTHE, UMR CNRS 7589 Sorbonne Universités, UPMC Paris 6, 75005 Paris, France \\ ${ }^{2}$ Albert Einstein Center, Institute for Theoretical Physics, Bern University, Sidlerstrasse 5, CH-3012 Bern
}

\begin{abstract}
We discuss possible connections between several scales in particle physics and cosmology, such the the electroweak, inflation, dark energy and Planck scales. We then describe the phenomenology of a model of supersymmetry breaking in the presence of a tiny (tunable) positive cosmological constant. The model is coupled to the MSSM, leading to calculable soft supersymmetry breaking masses and a distinct low energy phenomenology that allows to differentiate it from other models of supersymmetry breaking and mediation mechanisms.
\end{abstract}

\section{Introduction}

If String Theory is a fundamental theory of Nature and not just a tool for studying systems with strongly coupled dynamics, it should be able to describe at the same time particle physics and cosmology, which are phenomena that involve very different scales from the microscopic four-dimensional (4d) quantum gravity length of $10^{-33} \mathrm{~cm}$ to large macroscopic distances of the size of the observable Universe $\sim 10^{28} \mathrm{~cm}$ spanned a region of about 60 orders of magnitude. In particular, besides the $4 \mathrm{~d}$ Planck mass, there are three very different scales with very different physics corresponding to the electroweak, dark energy and inflation. These scales might be related via the scale of the underlying fundamental theory, such as string theory, or they might be independent in the sense that their origin could be based on different and independent dynamics. An example of the former constrained and more predictive possibility is provided by $\mathrm{TeV}$ strings with a fundamental scale at low energies due for instance to large extra dimensions transverse to a four-dimensional braneworld forming our Universe [1]. In this case, the $4 \mathrm{~d}$ Planck mass is emergent from the fundamental string scale and inflation should also happen around the same scale [2]. We will first review this possibility, focussing on its compatibility with cosmological observations.

We will then adopt a second more conservative approach, assuming that all three scales have an independent dynamical origin. Moreover, we will assume the presence of low energy supersymmetry that allows for an elegant solution of the mass hierarchy problem, a unification of fundamental forces as indicated by low energy data and a natural dark matter candidate due to an unbroken R-parity. The assumption of independent scales implies that supersymmetry breaking should be realized in a metastable de Sitter vacuum with an infinitesimally small (tunable) cosmological constant independent of the supersymmetry breaking scale that should be in the $\mathrm{TeV}$ region. In a recent work [3], we studied a simple $N=1$ supergravity model having this property and motivated by string theory.

ae-mail: ignatios.antoniadis@polytechnique.edu 
Besides the gravity multiplet, the minimal field content consists of a chiral multiplet with a shift symmetry promoted to a gauged R-symmetry using a vector multiplet. In the string theory context, the chiral multiplet can be identified with the string dilaton (or an appropriate compactification modulus) and the shift symmetry associated to the gauge invariance of a two-index antisymmetric tensor that can be dualized to a (pseudo)scalar. The shift symmetry fixes the form of the superpotential and the gauging allows for the presence of a Fayet-Iliopoulos (FI) term, leading to a supergravity action with two independent parameters that can be tuned so that the scalar potential possesses a metastable de Sitter minimum with a tiny vacuum energy (essentially the relative strength between the F- and D-term contributions). A third parameter fixes the Vacuum Expectation Value (VEV) of the string dilaton at the desired (phenomenologically) weak coupling regime. An important consistency constraint of our model is anomaly cancellation which has been studied in [5] and implies the existence of additional charged fields under the gauged R-symmetry.

In a more recent work [6], we analyzed a small variation of this model which is manifestly anomaly free without additional charged fields and allows to couple in a straight forward way a visible sector containing the minimal supersymmetric extension of the Standard Model (MSSM) and studied the mediation of supersymmetry breaking and its phenomenological consequences. It turns out that an additional 'hidden sector' field $z$ is needed to be added for the matter soft scalar masses to be nontachyonic; although this field participates in the supersymmetry breaking and is similar to the so-called Polonyi field, it does not modify the main properties of the metastable de Sitter (dS) vacuum. All soft scalar masses, as well as trilinear A-terms, are generated at the tree level and are universal under the assumption that matter kinetic terms are independent of the 'Polonyi' field, since matter fields are neutral under the shift symmetry and supersymmetry breaking is driven by a combination of the $U(1)$ D-term and the dilaton and $z$-field F-term. Alternatively, a way to avoid the tachyonic scalar masses without adding the extra field $z$ is to modify the matter kinetic terms by a dilaton dependent factor.

A main difference of the second analysis from the first work is that we use a field representation in which the gauged shift symmetry corresponds to an ordinary $U(1)$ and not an R-symmetry. The two representations differ by a Kähler transformation that leaves the classical supergravity action invariant. However, at the quantum level, there is a Green-Schwarz term generated that amounts an extra dilaton dependent contribution to the gauge kinetic terms needed to cancel the anomalies of the R-symmetry. This creates an apparent puzzle with the gaugino masses that vanish in the first representation but not in the latter. The resolution to the puzzle is based to the so called anomaly mediation contributions $[7,8]$ that explain precisely the above apparent discrepancy. It turns out that gaugino masses are generated at the quantum level and are thus suppressed compared to the scalar masses (and A-terms).

\section{Effective Planck mass and the inflation scale}

Low scale gravity with large extra dimensions is actually a particular case of a more general framework, where the UV cutoff is lower than the Planck scale due to the existence of a large number of particle species coupled to gravity [9]. Indeed, it was shown that the effective UV cutoff $M_{U V}$ is given by

$$
M_{U V}^{2}=M_{P}^{2} / N
$$

where the counting of independent species $N$ takes into account all particles which are not broad resonances, having a width less than their mass. For instance, in braneworld gravity with $n$ large extra dimensions of average size $R$, the particle species are the Kaluza-Klein (KK) excitations of the graviton (and other possible bulk modes), whose number at a given energy scale $E_{*}$ is given by

$$
N \simeq R^{n} E_{*}^{n} .
$$


Here, we work out the consequences of this scale dependence of the strength of gravity for inferring various quantities during inflation [2], which we take to be driven by a single field for economy of discussion and because the data doesn't compel us to consider otherwise [10]. As is to be expected, all dimensionless observables such as the amplitude and spectral properties of the perturbations are unaffected by the changing strength of gravity at inflationary energies. However, when one tries to infer an absolute energy scale for inflation, one finds that it is undetermined commensurate with (1) up to the unknown spectrum of universally coupled species between laboratory scales and the inflationary scale, the details of which we elaborate upon in the following.

According to the inflationary paradigm, the primordial perturbations observed in the CMB were created at horizon crossing during the quasi de Sitter (dS) phase of early accelerated expansion sourced by the inflaton field. Therefore all quantities that enter calculations of primordial correlation functions (which we subsequently relate to observables in the CMB) refer to quantities at the scale at which inflation occurred. We denote all quantities measured at the scale of inflation with a starred subscript. The dominant contribution to the temperature anisotropies comes from adiabatic perturbations ${ }^{1}$ sourced by the comoving curvature perturbation $\mathcal{R}$, defined as the conformal factor of the 3-metric $h_{i j}$ in comoving gauge:

$$
h_{i j}(t, x)=a^{2}(t) e^{2 \mathcal{R}(t, x)} \hat{h}_{i j} ; \quad \hat{h}_{i j}:=\exp \left[\gamma_{i j}\right]
$$

with $\partial_{i} \gamma_{i j}=\gamma_{i i}=0$ defining transverse traceless graviton perturbations. The temperature anisotropies are characterized by the dimensionless power spectrum for $\mathcal{R}$, whose amplitude is given by

$$
\mathcal{P}_{\mathcal{R}}:=\frac{H_{*}^{2}}{8 \pi^{2} M_{*}^{2} \epsilon_{*}}=\mathcal{A} \times 10^{-10},
$$

where $\epsilon_{*}:=-\dot{H}_{*} / H_{*}^{2}, H_{*}$ being the Hubble factor during inflation. Given that $\mathcal{R}$ is conserved on superhorizon scales (in the absence of entropy perturbations), this immediately relates to the amplitude of the late time $\mathrm{CMB}$ anisotropies, which fixes $\mathcal{A} \sim 22.15$ [10]. The tensor anisotropies are characterized by the tensor power spectrum

$$
\mathcal{P}_{\gamma}:=2 \frac{H_{*}^{2}}{\pi^{2} M_{*}^{2}},
$$

Taking the ratio of the above with (4), we find the tensor to scalar ratio

$$
r_{*}:=\frac{\mathcal{P}_{\gamma}}{\mathcal{P}_{\mathcal{R}}}=16 \epsilon_{*}
$$

Therefore any determination of $r_{*}$, either through direct measurements of the stochastic background of primordial gravitational waves or through their secondary effects on the polarization of the CMB [11-13] allows us in principle to fix the scale of inflation:

$$
H_{*}=M_{*}\left(\frac{\pi^{2} \mathcal{A} r_{*}}{2 \cdot 10^{10}}\right)^{1 / 2}:=\Upsilon=1.05 \sqrt{r}_{*} \times 10^{-4}
$$

We see that any measurements of $r_{*}$ determines the scale of inflation up to our ignorance of the effective strength of gravity at the scale $H_{*}$, given by $M_{*} \sim \frac{M_{P}}{\sqrt{N}}$, where $N$ is the effective number of all universally coupled species up to the scale $H_{*}$ - whether they exist in the visible sector or in any hidden sector. Note that as one lowers the scale of strong gravity, the maximum reheating temperature

\footnotetext{
${ }^{1}$ In what follows, we assume that all of the extra species have sufficiently suppressed couplings to the inflaton during inflation (e.g. either through derivative couplings or as Planck suppressed interactions) so that isocurvature perturbations are not significantly generated. This is trivially true for hidden sector fields.
} 
$T_{i}$ is necessarily lowered as well, since it cannot be higher than the inflation scale. Conservatively, $T_{i}$ cannot be too far below the TeV scale without spoiling the standard scenarios of big bang cosmologyin particular, mechanisms for Leptogenesis and Baryogenesis which can occur no lower than the electroweak scale [14]. We note as a consistency check on the above considerations, that although additional species increase the strength of gravity, the ratio $H_{*}^{2} / M_{*}^{2}$ is independent of $N$ and is fixed by observable quantities. Therefore the effects of strong gravity are evidently negligible during inflation even if $M_{*}$ is much smaller than the macroscopic strength of gravity $M_{\mathrm{pl}}$. Hence inflationary dynamics, in particular the dynamics of adiabatic fluctuations remain weakly coupled independent of $N$ and the usual computation of adiabatic correlators can be implemented [15].

In the case of extra species as KK graviton modes, the fundamental higher-dimensional gravity scale (1) with $N$ given in (2) for $E_{*}=M_{U V}$ leads to the usual relation between the $4 \mathrm{~d}$ and $(4+n) \mathrm{d}$ Planck scales

$$
M_{P}^{2}=M_{U V}^{2+n} R^{n} .
$$

On the other hand, during inflation $N$ counts all KK states with mass less than the Hubble scale $H_{*}$ :

$$
N=\left(H_{*} R\right)^{n},
$$

and the effective gravity scale becomes

$$
M_{*}=M_{P} / \sqrt{N}=M_{U V}\left(M_{U V} / H\right)^{n / 2},
$$

where we used the relations (8) and (10). Equation (7) then yields:

$$
H_{*}=M_{*} \Upsilon=M_{U V}\left(M_{U V} / H\right)^{n / 2} \Upsilon \Rightarrow M_{U V} \Upsilon^{2 /(n+2)},
$$

where we used eq. (10). It follows that $H_{*}$ is one to three orders of magnitude below the fundamental gravity scale $M_{U V}$ for the range $0.001 \lesssim r_{*} \lesssim 0.1$. The ratio $H_{*} / M_{*}$ is of course fixed by (7). The inflation scale $H_{*}$ can then be as low as the weak scale in low scale gravity models with large extra dimensions, consistently with observations.

In the following, we assume that the electroweak, inflation, gravity and dark energy scales have an independent dynamical origin and examine the corresponding conditions to the microscopic theories. More precisely, we address the question of supersymmetry breaking in dS space with an infinitesimal (tunable) cosmological constant.

\section{Conventions}

Throughout this paper we use the conventions of [16]. A supergravity theory is specified (up to ChernSimons terms) by a Kähler potential $\mathcal{K}$, a superpotential $W$, and the gauge kinetic functions $f_{A B}(z)$. The chiral multiplets $z^{\alpha}, \chi^{\alpha}$ are enumerated by the index $\alpha$ and the indices $A, B$ indicate the different gauge groups. Classically, a supergravity theory is invariant under Kähler tranformations, viz.

$$
\begin{aligned}
\mathcal{K}(z, \bar{z}) & \longrightarrow \mathcal{K}(z, \bar{z})+J(z)+\bar{J}(\bar{z}), \\
W(z) & \longrightarrow e^{-k^{2} J(z)} W(z),
\end{aligned}
$$

where $\kappa$ is the inverse of the reduced Planck mass, $m_{p}=\kappa^{-1}=2.4 \times 10^{15} \mathrm{TeV}$. The gauge transformations of chiral multiplet scalars are given by holomorphic Killing vectors, i.e. $\delta z^{\alpha}=\theta^{A} k_{A}^{\alpha}(z)$, where $\theta^{A}$ is the gauge parameter of the gauge group $A$. The Kähler potential and superpotential need not be invariant under this gauge transformation, but can change by a Kähler transformation

$$
\delta \mathcal{K}=\theta^{A}\left[r_{A}(z)+\bar{r}_{A}(\bar{z})\right],
$$


provided that the gauge transformation of the superpotential satisfies $\delta W=-\theta^{A} \kappa^{2} r_{A}(z) W$. One then has from $\delta W=W_{\alpha} \delta z^{\alpha}$

$$
W_{\alpha} k_{A}^{\alpha}=-\kappa^{2} r_{A} W
$$

where $W_{\alpha}=\partial_{\alpha} W$ and $\alpha$ labels the chiral multiplets. The supergravity theory can then be described by a gauge invariant function

$$
\mathcal{G}=\kappa^{2} \mathcal{K}+\log \left(\kappa^{6} W \bar{W}\right) .
$$

The scalar potential is given by

$$
\begin{aligned}
V & =V_{F}+V_{D} \\
V_{F} & =e^{\kappa^{2} \mathcal{K}}\left(-3 \kappa^{2} W \bar{W}+\nabla_{\alpha} W g^{\alpha \bar{\beta}} \bar{\nabla}_{\bar{\beta}} \bar{W}\right) \\
V_{D} & =\frac{1}{2}(\operatorname{Re} f)^{-1 A B} \mathcal{P}_{A} \mathcal{P}_{B},
\end{aligned}
$$

where W appears with its Kähler covariant derivative

$$
\nabla_{\alpha} W=\partial_{\alpha} W(z)+\kappa^{2}\left(\partial_{\alpha} \mathcal{K}\right) W(z)
$$

The moment maps $\mathcal{P}_{A}$ are given by

$$
\mathcal{P}_{A}=i\left(k_{A}^{\alpha} \partial_{\alpha} \mathcal{K}-r_{A}\right) .
$$

In this paper we will be concerned with theories having a gauged R-symmetry, for which $r_{A}(z)$ is given by an imaginary constant $r_{A}(z)=i \kappa^{-2} \xi$. In this case, $\kappa^{-2} \xi$ is a Fayet-Iliopoulos [17] constant parameter.

\section{The model}

The starting point is a chiral multiplet $S$ and a vector multiplet associated with a shift symmetry of the scalar component $s$ of the chiral multiplet $S$

$$
\delta s=-i c \theta
$$

and a string-inspired Kähler potential of the form $-p \log (s+\bar{s})$. The most general superpotential is either a constant $W=\kappa^{-3} a$ or an exponential superpotential $W=\kappa^{-3} a e^{b s}$ (where $a$ and $b$ are constants). A constant superpotential is (obviously) invariant under the shift symmetry, while an exponential superpotential transforms as $W \rightarrow W e^{-i b c \theta}$, as in eq. (14). In this case the shift symmetry becomes a gauged R-symmetry and the scalar potential contains a Fayet-Iliopoulos term. Note however that by performing a Kähler transformation (12) with $J=\kappa^{-2} b s$, the model can be recast into a constant superpotential at the cost of introducing a linear term in the Kähler potential $\delta K=b(s+\bar{s})$. Even though in this representation, the shift symmetry is not an R-symmetry, we will still refer to it as $U(1)_{R}$. The most general gauge kinetic function has a constant term and a term linear in $s, f(s)=\delta+\beta s$.

To summarise, ${ }^{2}$

$$
\begin{aligned}
\mathcal{K}(s, \bar{s}) & =-p \log (s+\bar{s})+b(s+\bar{s}), \\
W(s) & =a, \\
f(s) & =\delta+\beta s,
\end{aligned}
$$

\footnotetext{
${ }^{2}$ In superfields the shift symmetry (19) is given by $\delta S=-i c \Lambda$, where $\Lambda$ is the superfield generalization of the gauge parameter. The gauge invariant Kähler potential is then given by $\mathcal{K}(S, \bar{S})=-p \kappa^{-2} \log \left(S+\bar{S}+c V_{R}\right)+\kappa^{-2} b\left(S+\bar{S}+c V_{R}\right)$, where $V_{R}$ is the gauge superfield of the shift symmetry.
} 
where we have set the mass units $\kappa=1$. The constants $a$ and $b$ together with the constant $c$ in eq. (19) can be tuned to allow for an infinitesimally small cosmological constant and a TeV gravitino mass. For $b>0$, there always exists a supersymmetric AdS (anti-de Sitter) vacuum at $\langle s+\bar{s}\rangle=b / p$, while for $b=0$ (and $p<3$ ) there is an AdS vacuum with broken supersymmetry. We therefore focus on $b<0$. In the context of string theory, $S$ can be identified with a compactification modulus or the universal dilaton and (for negative $b$ ) the exponential superpotential may be generated by nonperturbative effects.

The scalar potential is given by:

$$
\begin{aligned}
V & =V_{F}+V_{D} \\
V_{F} & =a^{2} e^{\frac{b}{l}} l^{p-2}\left\{\frac{1}{p}(p l-b)^{2}-3 l^{2}\right\} \quad l=1 /(s+\bar{s}) \\
V_{D} & =c^{2} \frac{l}{\beta+2 \delta l}(p l-b)^{2}
\end{aligned}
$$

In the case where $S$ is the string dilaton, $V_{D}$ can be identified as the contribution of a magnetized D-brane, while $V_{F}$ for $b=0$ and $p=2$ coincides with the tree-level dilaton potential obtained by considering string theory away its critical dimension [18]. For $p \geq 3$ the scalar potential $V$ is positive and monotonically decreasing, while for $p<3$, its F-term part $V_{F}$ is unbounded from below when $s+\bar{s} \rightarrow 0$. On the other hand, the D-term part of the scalar potential $V_{D}$ is positive and diverges when $s+\bar{s} \rightarrow 0$ and for various values for the parameters an (infinitesimally small) positive (local) minimum of the potential can be found.

If we restrict ourselves to integer $p$, tunability of the vacuum energy restricts $p=2$ or $p=1$ when $f(s)=s$, or $p=1$ when the gauge kinetic function is constant. For $p=2$ and $f(s)=s$, the minimization of $V$ yields:

$$
\begin{aligned}
b / l & =\alpha \approx-0.183268, \quad p=2 \\
\frac{a^{2}}{b c^{2}} & =A_{2}(\alpha)+B_{2}(\alpha) \frac{\Lambda}{b^{3} c^{2}} \approx-50.6602+O(\Lambda),
\end{aligned}
$$

where $\Lambda$ is the value of $V$ at the minimum (i.e. the cosmological constant), $\alpha$ is the negative root of the polynomial $-x^{5}+7 x^{4}-10 x^{3}-22 x^{2}+40 x+8$ compatible with (23) for $\Lambda=0$ and $A_{2}(\alpha), B_{2}(\alpha)$ are given by

$$
A_{2}(\alpha)=2 e^{-\alpha} \frac{-4+4 \alpha-\alpha^{2}}{\alpha^{3}-4 \alpha^{2}-2 \alpha} \quad ; \quad B_{2}(\alpha)=2 \frac{\alpha^{2} e^{-\alpha}}{\alpha^{2}-4 \alpha-2}
$$

It follows that by carefully tuning $a$ and $c, \Lambda$ can be made positive and arbitrarily small independently of the supersymmetry breaking scale. A plot of the scalar potential for certain values of the parameters is shown in figure 1 .

At the minimum of the scalar potential, for nonzero $a$ and $b<0$, supersymmetry is broken by expectation values of both an F and D-term. Indeed the F-term and D-term contributions to the scalar potential are

$$
\begin{aligned}
\left.V_{F}\right|_{s+\bar{s}=\frac{\alpha}{b}} & =\frac{1}{2} a^{2} b^{2} e^{\alpha}\left(1-\frac{2}{\alpha}\right)^{2}>0, \\
\left.V_{D}\right|_{s+\bar{s}=\frac{\alpha}{b}} & =\frac{b^{3} c^{2}}{\alpha}\left(1-\frac{2}{\alpha}\right)^{2}>0 .
\end{aligned}
$$

The gravitino mass term is given by

$$
\left(m_{3 / 2}\right)^{2}=e^{\mathcal{G}}=\frac{a^{2} b^{2}}{\alpha^{2}} e^{\alpha} .
$$




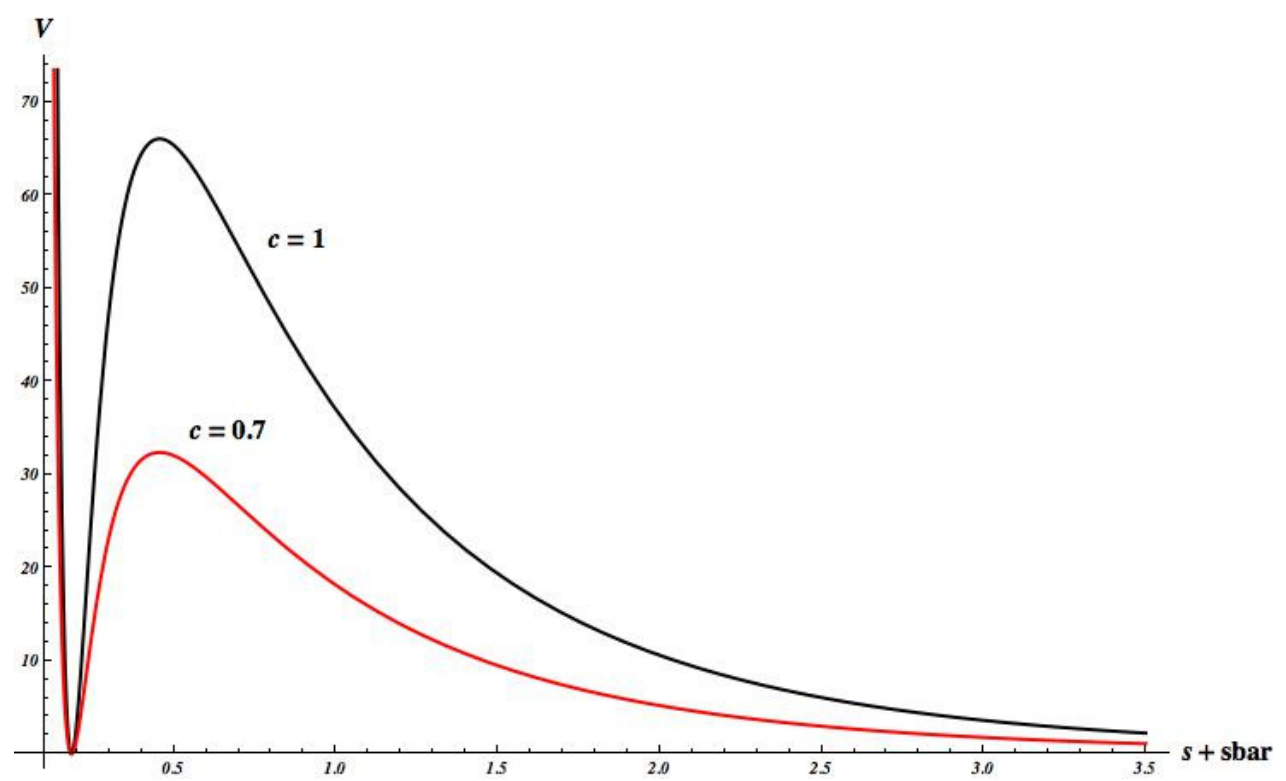

Figure 1. A plot of the scalar potential for $p=2, b=-1, \delta=0, \beta=1$ and $a$ given by equation (23) for $c=1$ (black curve) and $c=0.7$ (red curve).

Due to the Stueckelberg coupling, the imaginary part of $s$ (the axion) gets eaten by the gauge field, which acquires a mass. On the other hand, the Goldstino, which is a linear combination of the fermion of the chiral multiplet $\chi$ and the gaugino $\lambda$ gets eaten by the gravitino. As a result, the physical spectrum of the theory consists (besides the graviton) of a massive scalar, namely the dilaton, a Majorana fermion, a massive gauge field and a massive gravitino. All the masses are of the same order of magnitude as the gravitino mass, proportional to the same constant $a$ (or $c$ related by eq. (23) where $b$ is fixed by eq. (22)), which is a free parameter of the model. Thus, they vanish in the same way in the supersymmetric limit $a \rightarrow 0$.

The local dS minimum is metastable since it can tunnel to the supersymmetric ground state at infinity in the $s$-field space (zero coupling). It turns out however that it is extremely long lived for realistic perturbative values of the gauge coupling $l \simeq 0.02$ and $\mathrm{TeV}$ gravitino mass and, thus, practically stable; its decay rate is [5]:

$$
\Gamma \sim e^{-B} \quad \text { with } \quad B \approx 10^{300} .
$$

\section{Coupling a visible sector}

The guideline to construct a realistic model keeping the properties of the toy model described above is to assume that matter fields are invariant under the shift symmetry (19) and do not participate in the supersymmetry breaking. In the simplest case of a canonical Kähler potential, MSSM-like fields $\phi$ can then be added as:

$$
\begin{aligned}
\mathcal{K} & =-\kappa^{-2} \log (s+\bar{s})+\kappa^{-2} b(s+\bar{s})+\sum \varphi \bar{\varphi}, \\
W & =\kappa^{-3} a+W_{M S S M},
\end{aligned}
$$


where $W_{M S S M}(\phi)$ is the usual MSSM superpotential. The squared soft scalar masses of such a model can be shown to be positive and close to the square of the gravitino mass $\left(\mathrm{TeV}^{2}\right)$. On the other hand, for a gauge kinetic function with a linear term in $s, \beta \neq 0$ in eq. (20), the Lagrangian is not invariant under the shift symmetry

$$
\delta \mathcal{L}=-\theta \frac{\beta c}{8} \epsilon^{\mu \nu \rho \sigma} F_{\mu \nu} F_{\rho \sigma} .
$$

and its variation should be canceled. As explained in Ref. [5], in the 'frame' with an exponential superpotential the R-charges of the fermions in the model can give an anomalous contribution to the Lagrangian. In this case the 'Green-Schwarz' term $\operatorname{Im} s F \tilde{F}$ can cancel quantum anomalies. However as shown in [5], with the minimal MSSM spectrum, the presence of this term requires the existence of additional fields in the theory charged under the shift symmetry.

Instead, to avoid the discussion of anomalies, we focus on models with a constant gauge kinetic function. In this case the only (integer) possibility ${ }^{3}$ is $p=1$. The scalar potential is given by (21) with $\beta=0, \delta=p=1$. The minimization yields to equations similar to (22), (23) and (24) with a different value of $\alpha$ and functions $A_{1}$ and $B_{1}$ given by:

$$
\begin{aligned}
b\langle s+\bar{s}\rangle & =\alpha \approx-0.233153 \\
\frac{b c^{2}}{a^{2}} & =A_{1}(\alpha)+B_{1}(\alpha) \frac{\Lambda}{a^{2} b} \approx-0.359291+O(\Lambda) \\
A_{1}(\alpha) & =2 e^{\alpha} \alpha \frac{3-(\alpha-1)^{2}}{(\alpha-1)^{2}} \quad, \quad B_{1}(\alpha)=\frac{2 \alpha^{2}}{(\alpha-1)^{2}},
\end{aligned}
$$

where $\alpha$ is the negative root of $-3+(\alpha-1)^{2}\left(2-\alpha^{2} / 2\right)=0$ close to -0.23 , compatible with the second constraint for $\Lambda=0$. However, this model suffers from tachyonic soft masses when it is coupled to the MSSM, as in (28). To circumvent this problem, one can add an extra hidden sector field which contributes to (F-term) supersymmetry breaking. Alternatively, the problem of tachyonic soft masses can also be solved if one allows for a non-canonical Kähler potential in the visible sector, which gives an additional contribution to the masses through the D-term.

Let us discuss first the addition of an extra hidden sector field $z$ (similar to the so-called Polonyi field [19]). The Kähler potential, superpotential and gauge kinetic function are given by

$$
\begin{aligned}
\mathcal{K} & =-\kappa^{-2} \log (s+\bar{s})+\kappa^{-2} b(s+\bar{s})+z \bar{z}+\sum \varphi \bar{\varphi}, \\
W & =\kappa^{-3} a(1+\gamma \kappa z)+W_{\mathrm{MSSM}}(\varphi), \\
f(s) & =1, \quad f_{A}=1 / g_{A}^{2},
\end{aligned}
$$

where $A$ labels the Standard Model gauge group factors and $\gamma$ is an additional constant parameter. The existence of a tunable dS vacuum with supersymmetry breaking and non-tachyonic scalar masses implies that $\gamma$ must be in a narrow region:

$$
0.5 \lesssim \gamma \lesssim 1.7
$$

In the above range of $\gamma$ the main properties of the toy model described in the previous section remain, while Re $z$ and its F-auxiliary component acquire non vanishing VEVs. All MSSM soft scalar masses

\footnotetext{
${ }^{3}$ If $f(s)$ is constant, the leading contribution to $V_{D}$ when $s+\bar{s} \rightarrow 0$ is proportional to $1 /(s+\bar{s})^{2}$, while the leading contribution to $V_{F}$ is proportional to $1 /(s+\bar{s})^{p}$. It follows that $p<2$; if $p>2$, the potential is unbounded from below, while if $p=2$, the potential is either positive and monotonically decreasing or unbounded from below when $s+\bar{s} \rightarrow 0$ depending on the values of the parameters.
} 
are then equal to a universal value $m_{0}$ of the order of the gravitino mass, while the $B_{0}$ Higgs mixing parameter is also of the same order:

$$
\begin{aligned}
& m_{0}^{2}=m_{3 / 2}^{2}\left[\left(\sigma_{s}+1\right)+\frac{(\gamma+t+\gamma t)^{2}}{(1+\gamma t)^{2}}\right], \\
& A_{0}=m_{3 / 2}\left[\left(\sigma_{s}+3\right)+t \frac{\left(\gamma+t+\gamma t^{2}\right)}{1+\gamma t}\right], \\
& B_{0}=m_{3 / 2}\left[\left(\sigma_{s}+2\right)+t \frac{\left(\gamma+t+\gamma t^{2}\right)}{(1+\gamma t)}\right],
\end{aligned}
$$

where $\sigma_{s}=-3+(\alpha-1)^{2}$ with $\alpha$ and $t \equiv\langle\operatorname{Re} z\rangle$ determined by the minimization conditions as functions of $\gamma$. Also, $A_{0}$ is the soft trilinear scalar coupling in the standard notation, satisfying the relation [20]

$$
A_{0}=B_{0}+m_{3 / 2} \text {. }
$$

On the other hand, the gaugino masses appear to vanish at tree-level since the gauge kinetic functions are constants (see (31)). However, as mentioned in Section 4, this model is classically equivalent to the theory ${ }^{4}$

$$
\begin{aligned}
\mathcal{K} & =-\kappa^{-2} \log (s+\bar{s})+z \bar{z}+\sum_{\alpha} \varphi \bar{\varphi}, \\
W & =\left(\kappa^{-3} a(1+z)+W_{\operatorname{MSSM}}(\varphi)\right) e^{b s},
\end{aligned}
$$

obtained by applying a Kähler transformation (12) with $J=-\kappa^{-2} b s$. All classical results remain the same, such as the expressions for the scalar potential and the soft scalar masses (33), but now the shift symmetry (19) of $s$ became a gauged R-symmetry since the superpotential transforms as $W \longrightarrow W e^{-i b c \theta}$. Therefore, all fermions (including the gauginos and the gravitino) transform ${ }^{5}$ as well under this $U(1)_{R}$, leading to cubic $U(1)_{R}^{3}$ and mixed $U(1) \times G_{\mathrm{MSSM}}$ anomalies. These anomalies are cancelled by a Green-Schwarz (GS) counter term that arises from a quantum correction to the gauge kinetic functions:

$$
f_{A}(s)=1 / g_{A}^{2}+\beta_{A} s \quad \text { with } \quad \beta_{A}=\frac{b}{8 \pi^{2}}\left(T_{R_{A}}-T_{G_{A}}\right),
$$

where $T_{G}$ is the Dynkin index of the adjoint representation, normalized to $N$ for $S U(N)$, and $T_{R}$ is the Dynkin index associated with the representation $R$ of dimension $d_{R}$, equal to $1 / 2$ for the $S U(N)$ fundamental. An implicit sum over all matter representations is understood. It follows that gaugino masses are non-vanishing in this representation, creating a puzzle on the quantum equivalence of the two classically equivalent representations. The answer to this puzzle is based on the fact that gaugino masses are present in both representations and are generated at one-loop level by an effect called Anomaly Mediation [7, 8]. Indeed, it has been argued that gaugino masses receive a one-loop contribution due to the super-Weyl-Kähler and sigma-model anomalies, given by [8]:

$$
M_{1 / 2}=-\frac{g^{2}}{16 \pi^{2}}\left[\left(3 T_{G}-T_{R}\right) m_{3 / 2}+\left(T_{G}-T_{R}\right) \mathcal{K}_{\alpha} F^{\alpha}+2 \frac{T_{R}}{d_{R}}\left(\left.\log \operatorname{det} \mathcal{K}\right|_{R}{ }^{\prime \prime}\right)_{, \alpha} F^{\alpha}\right] .
$$

The expectation value of the auxiliary field $F^{\alpha}$, evaluated in the Einstein frame is given by

$$
F^{\alpha}=-e^{\kappa^{2} \mathcal{K} / 2} g^{\alpha \bar{\beta}} \bar{\nabla}_{\bar{\beta}} \bar{W}
$$

\footnotetext{
${ }^{4}$ This statement is only true for supergravity theories with a non-vanishing superpotential where everything can be defined in terms of a gauge invariant function $G=\kappa^{2} \mathcal{K}+\log \left(\kappa^{6} W \bar{W}\right)[21]$.

${ }^{5}$ The chiral fermions, the gauginos and the gravitino carry a charge $b c / 2,-b c / 2$ and $-b c / 2$ respectively.
} 
Clearly, for the Kähler potential (31) or (35) the last term in eq. (37) vanishes. However, the second term survives due to the presence of Planck scale VEVs for the hidden sector fields $s$ and $z$. Since the Kähler potential between the two representations differs by a linear term $b(s+\bar{s})$, the contribution of the second term in eq. (37) differs by a factor

$$
\delta m_{A}=\frac{g_{A}^{2}}{16 \pi^{2}}\left(T_{G}-T_{R}\right) b e^{\kappa^{2} \mathcal{K} / 2} g^{\alpha \bar{\beta}} \bar{\nabla}_{\bar{\beta}} \bar{W}
$$

which exactly coincides with the 'direct' contribution to the gaugino masses due to the field dependent gauge kinetic function (36) (taking into account a rescaling proportional to $g_{A}^{2}$ due to the non-canonical kinetic terms).

We conclude that even though the models (31) and (35) differ by a (classical) Kähler transformation, they generate the same gaugino masses at one-loop. While the one-loop gaugino masses for the model (31) are generated entirely by eq. (37), the gaugino masses for the model (35) after a Kähler transformation have a contribution from eq. (37) as well as from a field dependent gauge kinetic term whose presence is necessary to cancel the mixed $U(1)_{R} \times G$ anomalies due to the fact that the extra $U(1)$ has become an R-symmetry giving an R-charge to all fermions in the theory. Using (37), one finds:

$$
M_{1 / 2}=-\frac{g^{2}}{16 \pi^{2}} m_{3 / 2}\left[\left(3 T_{G}-T_{R}\right)-\left(T_{G}-T_{R}\right)\left((\alpha-1)^{2}+t \frac{\gamma+t+\gamma t^{2}}{1+\gamma t}\right)\right] .
$$

For $U(1)_{Y}$ we have $T_{G}=0$ and $T_{R}=11$, for $S U(2)$ we have $T_{G}=2$ and $T_{R}=7$, and for $S U(3)$ we have $T_{G}=3$ and $T_{R}=6$, such that for the different gaugino masses this gives (in a self-explanatory notation):

$$
\begin{aligned}
& M_{1}=11 \frac{g_{Y}^{2}}{16 \pi^{2}} m_{3 / 2}\left[1-(\alpha-1)^{2}-\frac{t(\gamma+t+\gamma t)}{1+\gamma t}\right], \\
& M_{2}=\frac{g_{2}^{2}}{16 \pi^{2}} m_{3 / 2}\left[1-5(\alpha-1)^{2}-5 \frac{t\left(\gamma+t+\gamma t^{2}\right)}{1+\gamma t}\right], \\
& M_{3}=-3 \frac{g_{3}^{2}}{16 \pi^{2}} m_{3 / 2}\left[1+(\alpha-1)^{2}+\frac{t\left(\gamma+t+\gamma t^{2}\right)}{1+\gamma t}\right] .
\end{aligned}
$$

\section{Phenomenology}

The results for the soft terms calculated in the previous section, evaluated for different values of the parameter $\gamma$ are summarised in Table 1. For every $\gamma$, the corresponding $t$ and $\alpha$ are calculated by imposing a vanishing cosmological constant at the minimum of the potential. The scalar soft masses and trilinear terms are then evaluated by eqs. (33) and the gaugino masses by eqs. (41). Note that the relation (34) is valid for all $\gamma$. We therefore do not list the parameter $B_{0}$.

In most phenomenological studies, $B_{0}$ is substituted for $\tan \beta$, the ratio between the two Higgs VEVs, as an input parameter for the renormalization group equations (RGE) that determine the low energy spectrum of the theory. Since $B_{0}$ is not a free parameter in our theory, but is fixed by eq. (34), this corresponds to a definite value of $\tan \beta$. For more details see [22] (and references therein). The corresponding $\tan \beta$ for a few particular choices for $\gamma$ are listed in the last two columns of table 1 for $\mu>0$ and $\mu<0$ respectively. No solutions were found for $\gamma \lesssim 1.1$, for both signs of $\mu$. The lighest supersymmetric particle (LSP) is given by the lightest neutralino and since $M_{1}<M_{2}$ (see table 1) the lightest neutralino is mostly Bino-like, in contrast with a typical mAMSB (minimal anomaly mediation supersymmetry breaking) scenario, where the lightest neutralino is mostly Wino-like [23]. 
Table 1. The soft terms (in terms of $m_{3 / 2}$ ) for various values of $\gamma$. If a solution to the RGE exists, the value of $\tan \beta$ is shown in the last columns for $\mu>0$ and $\mu<0$ respectively.

\begin{tabular}{|l|ll|lllll|rr|}
\hline$\gamma$ & $\mathrm{t}$ & $\alpha$ & $m_{0}$ & $A_{0}$ & $M_{1}$ & $M_{2}$ & $M_{3}$ & $\tan \beta(\mu>0)$ & $\tan \beta(\mu<0)$ \\
\hline 0.6 & 0.446 & -0.175 & 0.475 & 1.791 & 0.017 & 0.026 & 0.027 & & \\
1 & 0.409 & -0.134 & 0.719 & 1.719 & 0.015 & 0.025 & 0.026 & & \\
1.1 & 0.386 & -0.120 & 0.772 & 1.701 & 0.015 & 0.024 & 0.026 & 46 & 29 \\
1.4 & 0.390 & -0.068 & 0.905 & 1.646 & 0.014 & 0.023 & 0.026 & 40 & 23 \\
1.7 & 0.414 & -0.002 & 0.998 & 1.588 & 0.013 & 0.022 & 0.025 & 36 & 19 \\
\hline
\end{tabular}

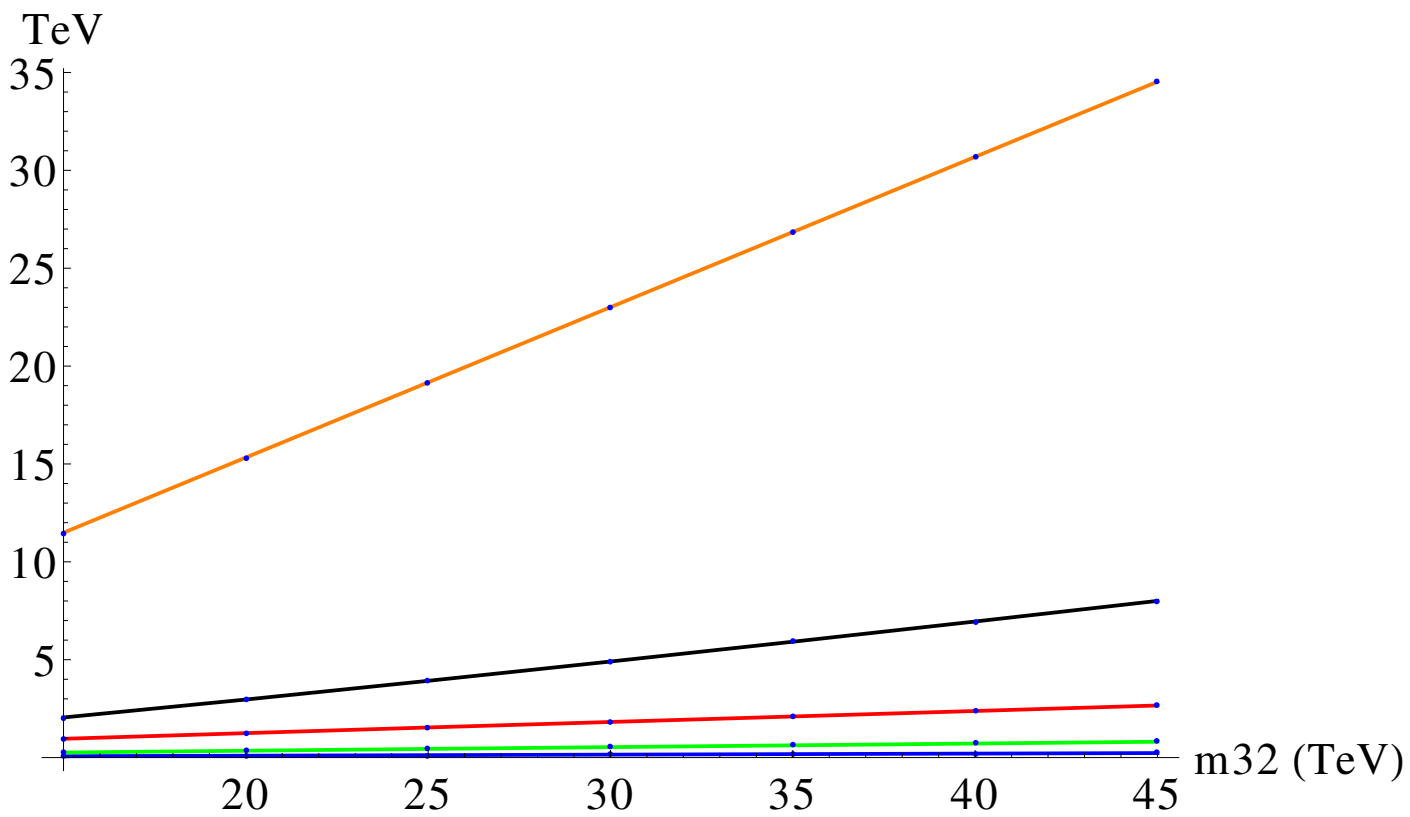

Figure 2. The masses (in TeV) of the sbottom (yellow), stop (black), gluino (red), lightest chargino (green) and lightest neutralino (blue) as a function of $m_{3 / 2}$ for $\gamma=1.1$ and for $\mu>0$. No solutions to the RGE were found when $m_{3 / 2} \gtrsim 45 \mathrm{TeV}$. The lower bound corresponds to a gluino mass of $1 \mathrm{TeV}$.

To get a lower bound on the stop mass, the sparticle spectrum is plotted in Figure 2 as a function of the gravitino mass for $\gamma=1.1$ and $\mu>0$ (for $\mu<0$ the bound is higher). The experimental limit on the gluino mass forces $m_{3 / 2} \gtrsim 15 \mathrm{TeV}$. In this limit the stop mass can be as low as $2 \mathrm{TeV}$. To conclude, the lower end mass spectrum consists of (very) light charginos (with a lightest chargino between 250 and $800 \mathrm{GeV}$ ) and neutralinos, with a mostly Bino-like neutralino as LSP $(80-230 \mathrm{GeV})$, which would distinguish this model from the mAMSB where the LSP is mostly Wino-like. These upper limits on the LSP and the lightest chargino imply that this model could in principle be excluded in the next LHC run. In order for the gluino to escape experimental bounds, the lower limit on the gravitino mass is about $15 \mathrm{TeV}$. The gluino mass is then between 1-3 TeV. This however forces the squark masses to be very high $(10-35 \mathrm{TeV})$, with the exception of the stop mass which can be relatively light $(2-15$ $\mathrm{TeV})$. 


\section{Non-canonical Kähler potential for the visible sector}

As mentioned already in Section 4, an alternative way to avoid tachyonic soft scalar masses for the MSSM fields in the model (28), instead of adding the extra Palonyi-type field $z$ in the hidden sector, is by introducing non-canonical kinetic terms for the MSSM fields, such as:

$$
\begin{aligned}
\mathcal{K} & =-\kappa^{-2} \log (s+\bar{s})+\kappa^{-2} b(s+\bar{s})+(s+\bar{s})^{-v} \sum \varphi \bar{\varphi}, \\
W & =\kappa^{-3} a+W_{M S S M}, \\
f(s) & =1, \quad f_{A}(s)=1 / g_{A}^{2},
\end{aligned}
$$

where $v$ is an additional parameter of the theory, with $v=1$ corresponding to the leading term in the Taylor expansion of $-\log (s+\bar{s}-\varphi \bar{\varphi})$. Since the visible sector fields appear only in the combination $\varphi \bar{\varphi}$, their VEVs vanish provided that the scalar soft masses squared are positive. Moreover, for vanishing visible sector VEVs, the scalar potential and is minimization remains the same as in eqs. (refbsalpha). Therefore, the non-canonical Kähler potential does not change the fact that the F-term contribution to the soft scalar masses squared is negative. On the other hand, the visible fields enter in the D-term scalar potential through the derivative of the Kähler potential with respect to $s$. Even though this has no effect on the ground state of the potential, the $\varphi$-dependence of the D-term scalar potential does result in an extra contribution to the scalar masses squared which become positive

$$
v>-\frac{e^{\alpha}\left(\sigma_{s}+1\right) \alpha}{A(\alpha)(1-\alpha)} \approx 2.6
$$

The soft MSSM scalar masses and trilinear couplings in this model are:

$$
\begin{aligned}
& m_{0}^{2}=\kappa^{2} a^{2}\left(\frac{b}{\alpha}\right)\left(e^{\alpha}\left(\sigma_{s}+1\right)+v \frac{A(\alpha)}{\alpha}(1-\alpha)\right) \\
& A_{0}=m_{3 / 2}(s+\bar{s})^{v / 2}\left(\sigma_{s}+3\right) \\
& B_{0}=m_{3 / 2}(s+\bar{s})^{v / 2}\left(\sigma_{s}+2\right)
\end{aligned}
$$

where $\sigma_{s}$ is defined as in (33), eq. (31) has been used to relate the constants $a$ and $c$, and corrections due to a small cosmological constant have been neglected. A field redefinition due to a non-canonical kinetic term $g_{\varphi \bar{\varphi}}=(s+\bar{s})^{-v}$ is also taken into account. The main phenomenological properties of this model are not expected to be different from the one we analyzed in section 6 with the parameter $v$ replacing $\gamma$. Gaugino masses are still generated at one-loop level while mSUGRA applies to the soft scalar sector. We therefore do not repeat the phenomenological analysis for this model.

\section{References}

[1] I. Antoniadis, N. Arkani-Hamed, S. Dimopoulos and G. R. Dvali, Phys. Lett. B 436 (1998) 257 [arXiv:hep-ph/9804398].

[2] I. Antoniadis and S. P. Patil, Eur. Phys. J. C75 (2015) 182 [arXiv:1410.8845 [hep-th]].

[3] I. Antoniadis and R. Knoops, Nucl. Phys. B 886 (2014) 43 [arXiv:1403.1534 [hep-th]].

[4] F. Catino, G. Villadoro and F. Zwirner, JHEP 1201 (2012) 002 [arXiv:1110.2174 [hep-th]]; G. Villadoro and F. Zwirner, Phys. Rev. Lett. 95 (2005) 231602 [hep-th/0508167].

[5] I. Antoniadis, D. M. Ghilencea and R. Knoops, JHEP 1502 (2015) 166 [arXiv:1412.4807 [hepth]].

[6] I. Antoniadis and R. Knoops, [arXiv:1507.06924 [hep-ph]]. 
[7] L. Randall and R. Sundrum, Nucl. Phys. B 557 (1999) 79 [hep-th/9810155]; G. F. Giudice, M. A. Luty, H. Murayama and R. Rattazzi, JHEP 9812 (1998) 027 [hep-ph/9810442].

[8] J. A. Bagger, T. Moroi and E. Poppitz, JHEP 0004 (2000) 009 [hep-th/9911029].

[9] G. Dvali, arXiv:0706.2050 [hep-th]; Int. J. Mod. Phys. A 25 (2010) 602 [arXiv:0806.3801 [hep-th]]; G. Dvali and M. Redi, Phys. Rev. D 77 (2008) 045027 [arXiv:0710.4344 [hep-th]]; R. Brustein, G. Dvali and G. Veneziano, JHEP 0910 (2009) 085 [arXiv:0907.5516 [hep-th]]; G. Dvali and C. Gomez, Phys. Lett. B 674 (2009) 303.

[10] P. A. R. Ade et al. [Planck Collaboration], Astron. Astrophys. (2014) [arXiv:1303.5076 [astroph.CO]]; arXiv:1303.5082 [astro-ph.CO].

[11] A. G. Polnarev, Sov. Astron. 29, 607 (1985).

[12] M. Kamionkowski, A. Kosowsky and A. Stebbins, Phys. Rev. Lett. 78, 2058 (1997) [astro$\mathrm{ph} / 9609132]$.

[13] U. Seljak and M. Zaldarriaga, Phys. Rev. Lett. 78, 2054 (1997) [astro-ph/9609169].

[14] M. Trodden, Rev. Mod. Phys. 71, 1463 (1999) [hep-ph/9803479]; S. Davidson, E. Nardi and Y. Nir, Phys. Rept. 466, 105 (2008) [arXiv:0802.2962 [hep-ph]].

[15] C. Cheung, P. Creminelli, A. L. Fitzpatrick, J. Kaplan and L. Senatore, JHEP 0803, 014 (2008) [arXiv:0709.0293 [hep-th]].

[16] D. Z. Freedman and A. Van Proeyen, Cambridge, UK: Cambridge Univ. Pr. (2012) 607 p.

[17] P. Fayet and J. Iliopoulos, Phys. Lett. B 51 (1974) 461; Phys. Lett. B 69 (1977) 489.

[18] I. Antoniadis, J. -P. Derendinger and T. Maillard, Nucl. Phys. B 808 (2009) 53 [arXiv:0804.1738 [hep-th]].

[19] J. Polonyi, Hungary Central Inst Res - KFKI-77-93 (77,REC.JUL 78) 5p.

[20] H. P. Nilles, Phys. Rept. 110 (1984) 1

[21] S. Ferrara, L. Girardello, T. Kugo and A. Van Proeyen, Nucl. Phys. B 223 (1983) 191

[22] J. R. Ellis, K. A. Olive, Y. Santoso and V. C. Spanos, Phys. Lett. B 573 (2003) 162 [hep$\mathrm{ph} / 0305212]$,

[23] T. Gherghetta, G. F. Giudice and J. D. Wells, Nucl. Phys. B 559 (1999) 27 [hep-ph/9904378]. 